A sharp threshold for rainbow connection in small-world networks

\author{
Y. Shang
}




\title{
A SHARP THRESHOLD FOR RAINBOW CONNECTION IN SMALL-WORLD NETWORKS
}

\author{
Y. SHANG \\ Received 30 March, 2011
}

\begin{abstract}
An edge-colored graph $G$ is rainbow connected if any two vertices are connected by a path whose edges have distinct colors. The rainbow connection of a connected graph $G$, denoted by $r c(G)$, is the smallest number of colors that are needed in order to make $G$ rainbow connected. We prove that $p=\sqrt{\ln n / n}$ is a sharp threshold function for the property $\operatorname{rc}(S(n, p, H)) \leq 2$ in the small-world networks. As by-products, our extension of the concept of independence in graph theory and generalized small-world network models are of independent interest.
\end{abstract}

2000 Mathematics Subject Classification: 05C82; 05C15; 05C40

Keywords: rainbow connection, edge coloring, small world, networks

\section{INTRODUCTION}

We utilize the terminology and notation of [19] in this letter. An interesting connectivity concept of a graph was recently introduced in [3] and has attracted attention of some researchers. An edge-colored graph $G$ is referred to as rainbow connected if any two vertices are connected by a path whose edges have distinct colors. A rainbow connected graph must be connected, and conversely, any connected graph has a trivial edge coloring that makes it rainbow connected. The rainbow connection of a connected graph $G$, denoted $r c(G)$, is the smallest number of colors that are needed in order to make $G$ rainbow connected.

An easy observation is that if $G$ has $n$ vertices then $\operatorname{rc}(G) \leq n-1$, since one may color the edges of a given spanning tree of $G$ with distinct colors, and color the remaining edges with one of the already used colors. It is also known that $\operatorname{rc}(G)=1$ if and only if $G$ is a complete graph, and that $r c(G)=n-1$ if and only if $G$ is a tree. Note that $r c(G) \geq \operatorname{diam}(G)$, where $\operatorname{diam}(G)$ denotes the diameter of $G$. The behavior of $r c(G)$ with respect to the minimum degree $\delta(G)$ has been dealt with in the work [2,13], of which a primary result is $\operatorname{rc}(G) \leq 20 n / \delta(G)$. Related concepts such as rainbow path [6], rainbow tree [5] and rainbow $k$-connectivity [4] have also been investigated recently.

A natural and intriguing direction to explore is the random graph scenarios [12,17]. Let $G(n, p)$ be the classical random graph with $n$ vertices and edge probability $p$. For 
a graph property $\mathcal{A}$, we say that $G(n, p)$ satisfies $\mathcal{A}$ almost surely if the probability that $G(n, p)$ satisfies $\mathcal{A}$ tends to 1 as $n$ tends to infinity. A function $f(n)$ is called a sharp threshold function for the property $\mathcal{A}$ if there are two positive constants $C$ and $c$ such that $G(n, p)$ satisfies $\mathcal{A}$ almost surely for $p \geq C f(n)$ and $G(n, p)$ almost surely does not satisfy $\mathcal{A}$ for $p \leq c f(n)$. A remarkable feature of random graphs is that all monotone graph properties have sharp thresholds (see e.g. $[1,10,11]$ ).

The parameter $r c(G)$ is monotone non-increasing in the sense that if we add an edge to $G$ we cannot increase its rainbow connection. The authors of [2] show that $p=\sqrt{\ln n / n}$ is a sharp threshold function for the property $r c(G(n, p)) \leq 2$. In this note, we propose a generalized small-world network model and explore the threshold of rainbow connection of it. The small-world network is a model with two important characteristics: the clustering effect and the small-world phenomenon, which was originally introduced by Watts and Strogatz [18] as a model of real world complex networks. It has since been the subject of considerable research interest within the physics community, see e.g. [7,14-16] and references therein.

The rest of the note is organized as follows. In Section 2, we present some necessary notions including the generalized small-world model and state our sharp threshold result. The proofs are given in Section 3.

\section{NOTIONS AND MAIN RESULT}

Watts-Strogatz (WS) rewiring model [18] and its variant Newman-Watts (NW) model [16] are classical small-world network models. The NW model can be regarded as the union of an Erdős-Rényi random graph $G(n, p)$ and a $2 k$-regular lattice. It is known that the NW model and the WS model are, essentially, the same. A natural extension would be to use a general sparse graph to replace the low-dimensional regular lattices.

Let $S(n, p, H)$ be a small-world network that is the union of a random graph $G(n, p)$ and a graph $H$ on $n$ vertices. Note that $S(n, p, H)$ is not necessarily connected when $H$ is not connected. When $H$ is a regular lattice, we then obtain the NW model.

Next, we need to extend the classical notion of independence in graph theory to distant $l$-independence. A subset $X$ of vertices in a graph $G$ is called distant $l$ independent for some $l \in \mathbb{N}$, if the distance between any two vertices in $V$ is larger than $l$. Thus, a distant 1 -independent set is independent in the classical sense. Recall that there is another generalization of independence, called $k$-independence $[8,9]$, which requires the induced subgraph has maximum degree less than $k$. The relative strength relationship of these three concepts can be described as follows:

$$
k \text {-independence }<\text { independence }<\text { distant } l \text {-independence. }
$$

Now we are on the stage to state our main result. 
Theorem 1. Let $H$ be a graph on $n$ vertices, which contains a distant 2-independent set of order $\Theta\left(n^{\varepsilon}\right)$ for some $\varepsilon>0$. For the small-world network $S(n, p, H), p=$ $\sqrt{\ln n / n}$ is a sharp threshold function for the property $\operatorname{rc}(S(n, p, H)) \leq 2$.

Clearly, a $2 k$-regular lattice with $k \ll n^{\alpha}$ for some $\alpha \in(0,1)$ serves as an eligible graph $H$ in Theorem 1. Therefore, the above result holds for both WS and NW models.

\section{Proof of Theorem 1}

In this section, we will provide a proof of Theorem 1 as per the reasoning in [2]. As mentioned in Section $1, r c(G) \geq 2$ for any non-complete graph $G$. The following lemma gives a sufficient condition for $\operatorname{rc}(G)=2$.

Lemma 1. ([2]) If $G$ is a non-complete graph on $n$ vertices and any two vertices of $G$ have at least $2 \ln n$ common neighbors, then $\operatorname{rc}(G)=2$.

Proof of Theorem 1. For the first part of the theorem, we need to prove that for a sufficiently large constant $C$, the small-world network $S(n, p, H)$ with $p=C \sqrt{\ln n / n}$ almost surely has $r c(G)=2$. Recall that $r c(G)$ is monotone non-increasing, we need only to prove this for the random graph $G(n, p)$. By Lemma 1, it suffices to show that almost surely any two vertices of $G(n, p)$ have at least $2 \ln n$ common neighbors.

Fix a pair of vertices $x, y$, and the probability that $z$ is a common neighbor of them is $C^{2} \ln n / n$. Let random variable $X$ represents the number of common neighbors of $x$ and $y$. Accordingly, we get $E X=(n-2)\left(C^{2} \ln n / n\right)$. By using the Chernoff bound (e.g. [12] pp.26), for large enough $C$, we have

$$
P(X<2 \ln n) \leq P\left(X<E X-\frac{C^{2} \ln n}{4}\right) \leq e^{-\frac{C^{2} \ln n}{32}}=o\left(n^{-2}\right) .
$$

Since there are $\left(\begin{array}{l}n \\ 2\end{array}\right)$ pairs of vertices in $G(n, p)$, the union bound readily yields the result.

For the other direction, it suffices to show that for a sufficiently small constant $c$, the small-world network $S(n, p, H)$ with $p=c \sqrt{\ln n / n}$ almost surely has $\operatorname{diam}(S(n, p, H)) \geq 3$. By the assumption in Theorem 1, fix a distant 2-independent set $X$ of order $\Theta\left(n^{\varepsilon}\right)$ for some $\varepsilon<1 / 4$ in $H$, and let $Y$ be the remaining $n-\Theta\left(n^{\varepsilon}\right)$ vertices. Let $\mathcal{A}$ be the event that $X$ induces an independent set in the small-world network $S(n, p, H)$. Let $\mathcal{B}$ be the event that there exists a pair of vertices $x, y \in X$ with no common neighbor in $Y$. Consequently, it suffices to prove that (i) $P(\mathcal{A}) \rightarrow 1$; and (ii) $P(\mathscr{B}) \rightarrow 1$, as $n \rightarrow \infty$.

For (i): For $c$ sufficiently small we obtain

$$
\begin{aligned}
P(\mathcal{A}) & =(1-p)^{\left(\begin{array}{c}
\Theta\left(n^{\varepsilon}\right) \\
2
\end{array}\right)}=(1-c \sqrt{\ln n / n})\left(\begin{array}{c}
\Theta\left(n^{\varepsilon}\right) \\
2
\end{array}\right) \\
& \sim e^{-\frac{c \sqrt{\ln n}}{\Theta\left(n^{\frac{1}{2}-2 \varepsilon}\right)}} \rightarrow 1,
\end{aligned}
$$


as $n \rightarrow \infty$, since $0<\varepsilon<1 / 4$.

For (ii): For a pair $x, y \in X$, the probability that $x, y$ have a common neighbor in $Y$ is shown to be given by

$$
1-\left(1-\frac{c^{2} \ln n}{n}\right)^{n-\Theta\left(n^{\varepsilon}\right)} \sim\left(1-n^{-c^{2}}\right) .
$$

Since the vertex set $X$ can be divided into $\Theta\left(n^{\varepsilon}\right) / 2=\Theta\left(n^{\varepsilon}\right)$ pairs, the probability that all $\Theta\left(n^{\varepsilon}\right)$ pairs have a common neighbor is

$$
1-P(\mathscr{B})=\left(1-\left(1-\frac{c^{2} \ln n}{n}\right)^{n-\Theta\left(n^{\varepsilon}\right)}\right)^{\Theta\left(n^{\varepsilon}\right)} \sim\left(1-n^{-c^{2}}\right)^{\Theta\left(n^{\varepsilon}\right)} \sim e^{-\frac{\Theta\left(n^{\varepsilon}\right)}{n^{2}}} .
$$

For sufficiently small $c$, the right hand side of (3.1) tends to zero, which thus completes the proof.

\section{REFERENCES}

[1] B. Bollobás and A. Thomason, "Threshold functions," Combinatorica, vol. 7, pp. 35-38, 1987.

[2] Y. Caro, A. Lev, Y. Roditty, Z. Tuza, and R. Yuster, "On rainbow connection,” Electron. J. Comb., vol. 15, no. 1, pp. 13, Research Paper R57, 2008.

[3] G. Chartrand, G. L. Johns, K. A. McKeon, and P. Zhang, "Rainbow connection in graphs," Math. Bohem., vol. 133, no. 1, pp. 85-98, 2008.

[4] G. Chartrand, G. L. Johns, K. A. McKeon, and P. Zhang, "The rainbow connectivity of a graph," Networks, vol. 54, no. 2, pp. 75-81, 2009.

[5] G. Chartrand, F. Okamoto, and P. Zhang, "Rainbow trees in graphs and generalized connectivity," Networks, vol. 55, no. 4, pp. 360-367, 2010.

[6] D. j. Dellamonica, C. Magnant, and D. M. Martin, "Rainbow paths," Discrete Math., vol. 310, no. 4, pp. 774-781, 2010.

[7] R. Durrett, Random graph dynamics, ser. Cambridge Series in Statistical and Probabilistic Mathematics. Cambridge: Cambridge University Press, 2007, vol. 20.

[8] J. F. Fink and M. S. Jacobson, "n-domination in graphs," in Graph theory with applications to algorithms and computer science, ser. Proc. 5th Int. Conf., Kalamazoo/Mich., 1984. New York: John Wiley \& Sons, 1985, pp. 283-300.

[9] J. F. Fink and M. S. Jacobson, "On n-domination, n-dependence and forbidden subgraphs," in Graph theory with applications to algorithms and computer science, ser. Proc. 5th Int. Conf., Kalamazoo/Mich. 1984. New York: John Wiley \& Sons, 1985, pp. 301-311.

[10] E. Friedgut, "Hunting for sharp thresholds," Random Struct. Algorithms, vol. 26, no. 1-2, pp. 37-51, 2005.

[11] E. Friedgut and G. Kalai, "Every monotone graph property has a sharp threshold," Proc. Am. Math. Soc., vol. 124, no. 10, pp. 2993-3002, 1996.

[12] S. Janson, T. Łuczak, and A. Ruciński, Random graphs, ser. Wiley-Interscience Series in Discrete Mathematics and Optimization. New York: Wiley, 2000.

[13] M. Krivelevich and R. Yuster, "The rainbow connection of a graph is (at most) reciprocal to its minimum degree," J. Graph Theory, vol. 63, no. 3, pp. 185-191, 2010.

[14] M. E. Newman, "Models of the small world," J. Stat. Phys., vol. 101, no. 3-4, pp. 819-841, 2000.

[15] M. E. J. Newman, C. Moore, and D. J. Watts, "Mean-field solution of the small-world network model," Phys. Rev. Lett., vol. 84, pp. 3201-3204, 2000.

[16] M. E. J. Newman and D. J. Watts, "Renormalization group analysis of the small-world network model," Phys. Lett., A, vol. 263, no. 4-6, pp. 341-346, 1999. 
[17] Y. Shang, "Sharp concentration of the rainbow connection of random graphs," Notes Number Theory Discrete Math., vol. 16, no. 4, pp. 25-28, 2010.

[18] D. J. Watts and S. H. Strogatz, "Collective dynamics of "small-world" networks," Nature, vol. 393, pp. 440-442, 1998.

[19] D. B. West, Introduction to graph theory. Prentice Hall, 2000.

$\mathrm{f}$

\section{Author's address}

\section{Y. Shang}

University of Texas at San Antonio, Institute for Cyber Security, San Antonio, TX 78249, USA

E-mail address: shylmath@hotmail.com 